\title{
Defining a prognostic criterion of orthopaedic complications development in patients with an injury of anterior cruciate ligament of knee joint
}

\author{
DOI: https://doi.org/10.5114/pq.2019.87596
}

\author{
Andrey Rusanov', Irina Roy', Olga Rusanova ${ }^{2}$ \\ ${ }^{1}$ Institute of Traumatology and Orthopaedics, National Academy of Medical Sciences of Ukraine, Kyiv, Ukraine \\ ${ }^{2}$ National University of Ukraine on Physical Education and Sport, Kyiv, Ukraine
}

\begin{abstract}
Introduction. Prognostic indices of orthopaedic implications development were investigated among patients with anterior cruciate ligament $(A C L)$ injuries on the basis of stabilography research during the functional period of rehabilitation.

Methods. The study included 52 randomly selected individuals with a complete ACL tear qualified for surgical reconstruction. The patients' age was 18-59 (average: $37.8 \pm 2.0$ ) years. All participants underwent rehabilitation treatment in the Institute of Traumatology and Orthopaedics, National Academy of Medical Sciences of Ukraine, and had surgeries in the Institute's clinics. The crural part of the graft was fixed with the assistance of either the RigidFix system or the Cross-Pin system, and the Biointrafix or Biosure Sync system was applied for the tibial part.

Results and conclusions. On the basis of the performed analysis of indices that characterize the functional condition of the injured limb of patients with an injury of ACL of knee joint at the functional stage of rehabilitation treatment, it is possible to identify a high and medium level of loading asymmetry in the lower extremities, decrease of electrobiological activity and muscle resistance ability during bending, stretching, adduction, and abduction, as the main prognostic criterion of orthopaedic complications development.

Key words: physical rehabilitation, trauma, anterior cruciate ligament, knee joint, recovery treatment
\end{abstract}

\section{Introduction}

Arthroscopic surgical procedures are of significant importance for the development of anterior cruciate ligament (ACL) injury treatment methods, and nowadays the most progressive method of ACL function renewal is reconstruction [1]. Such surgical interventions allow not only to keep the normal biomechanics of the knee joint, but also to prevent early development of posttraumatic osteoarthrosis of the knee [2].

The evaluation of $A C L$ reconstruction results includes a list of objective and subjective data, which are stemming immediately from the individual functional requests of a patient. Among the objective criteria, the request for the level of achieved stability, preserved movement amplitude, existence and degree of asymmetry of lower extremities load balancing are the important ones. Subjective measurement places its priorities on the feeling of persistence of the joint and pain severity syndrome [3. Within the limits of physiological stress after a successful ACL reconstruction, the joint should be painless and stable, with full movement amplitude preserved and full support ability renewed in the extremity which underwent the surgical procedure.

Very often, an insufficient stability of the joint, which is connected to tactical and technical defects of the reconstruction, is compensated by existing contracture in the early postsurgical period. Further on, owing to contracture, the deforming arthrosis and arthrofibrosis become more severe, which leads, in turn, to the patient being in pain. The main causes of contracture are a non-isometric graft, lengthy postsurgical immobilization, and inadequate rehabilitation.

The aim of the research was to assess the factors that determine the functional status of the knee joint in patients 20 weeks after surgery.

\section{Subjects and methods}

\section{Participants}

The study included 52 randomly selected individuals with a complete ACL tear who were qualified for surgical reconstruction. The age of the participants was 18-59 (average: $37.8 \pm 2.0$ ) years.

Among the exclusion criteria, there were partial ruptures of the ACL, combined injuries of the ACL and meniscus, and $A C L$ and cartilage damage, identified by ultrasound and magnetic resonance imaging (MRI).

In the process of the research, we systematized and consolidated data from 52 medical records of patients treated in the Institute of Traumatology and Orthopaedics, National Academy of Medical Sciences of Ukraine during the period of 2013-2016. The patients, without temporary contraindications for arthroscopic operative intervention, were examined by using instrumental methods of research. The examination was performed within different periods of recovery treatment.

Overall, 21 participants (the main group) underwent rehabilitation in accordance with the proposed program after ACL reconstruction; in 31 patients (control group), the traditional

Correspondence address: Olga Rusanova, Sport and Management Faculty, National University of Ukraine on Physical Education and Sport, Fizkul'tury St, 1, Kyiv-150, Ukraine, 03150, e-mail: rusanova_2013@mail.ua 
program of physical rehabilitation was implemented. The results registered at the preliminary stage of the research showed no statistically significant differences between the examined indicators and the age of the patients among the control group and the main group.

All the subjects (52 people) underwent rehabilitation treatment in the rehabilitation department of the Institute of Traumatology and Orthopaedics, National Academy of Medical Sciences of Ukraine and were operated on in the hospitals of the Institute. The femoral end of the transplant was fixed with the help of the RigidFix or Cross-Pin systems, and the tibial end - by using the Biointrafix or Biosure Sync systems. Medication was applied in accordance with the prescriptions, when medically required.

\section{Procedure}

To achieve the set target in this study, the following methods were used: theoretical analysis of data from the scientific and methodological literature; pedagogical; clinical (examination, anamnesis collection); instrumental methods of research (anthropometric measurements, goniomenty, dynamomentry, electromyography, stabilography); and methods of mathematical data processing.

All the materials for the work were obtained during studies based on the State Enterprise 'Institute of Traumatology and Orthopaedics, National Academy of Medical Sciences of Ukraine'. The algorithm of the complex diagnostics for acute ACL injuries, created in the Institute, was applied to the patients having corresponding claims; the algorithm implied clinical, functional, X-ray, ultrasound, and MRI examination.

In all the hospitalized patients, the following procedures were applied: definition of the patient's claims, anamnesis; clinical examination, palpation; examination of the injured joint function, evaluation of instability level, presence of block, synovitis, infiltration, muscular atrophy, etc.; joint immobilization with the help of a semi-rigid brace (if necessary); X-ray, ultrasound, MRI (if possible) examination of knee joint. The obtained data were recorded in the patients' medical history.

During the clinical examination and anamnesis collection, all participants were asked about the reason of turning to the rehabilitation department; the intensity of pain syndrome (the Visual Analogue Scale of pain was applied); their claims on pain syndrome during active movements, passive movements, and at rest; instability of knee joint (measured by the Lachman test and drawer test; furthermore, quantitative measurements of instability were performed in accordance with the criteria of the American Association of Arthroscopy. The results of anthropometric measurements and goniometry of the injured and intact limbs were also registered in the patients' medical history.

\section{Statistical analysis}

The empirical material was processed with mathematical statistics methods. Statistical analyses were performed with the Statistical Package for the Social Sciences (SPSS) 10.0. The following methods of mathematical statistics were applied: descriptive statistics, selective method, Shapiro-Wilk normality test, Student's $t$-test, non-parametric Mann-Whitney test, factor analysis. Methods of descriptive analysis were used, including tabular presentation of separate variables, calculation of mean arithmetic value $(\bar{x})$, standard deviation $(S D)$, and indices of individual differences - coefficients of variation $(\mathrm{CV})$. The sample data for normality were tested with the normal distribution formula, Shapiro-Wilk test. To deter- mine the statistical significance of the differences between the samples of normal distribution, the Student's test was used. The level of $p=0.05$ (the probability of error) was assumed statistically significant. The informativeness of the tests and indicators was recorded, evaluated under standard conditions of measurement.

\section{Ethical approval}

The research related to human use has complied with all the relevant national regulations and institutional policies, has followed the tenets of the Declaration of Helsinki, and has been approved by the authors' institutional review board or an equivalent committee.

\section{Informed consent}

Informed consent has been obtained from all individuals included in this study.

\section{Results}

The indicators of movement range when bending the injured limb in its knee joint were quite low - with the normal movement range equal to $140^{\circ}$, among patients with $\mathrm{ACL}$ knee joint injuries it showed $102.91 \pm 4.41^{\circ}(\bar{x} \pm S D)$, which is $37.09^{\circ}$ less than norm and corresponds to $73.5 \%$ of the normal movement range $(p<0.05)$.

Because of the knee joint function deficiency, contracture in the damaged joint, pain sensations, and muscle hypotrophy in the injured limb, the patients manifested a decrease of muscle strength in the injured limb as compared with the intact limb $(p<0.05)$ while making the following movements: bending in knee joint (up to $36.43 \%$ ) and straightening (up to $32.13 \%)$.

Following the results of superficial electromyography examination, the estimation of the bioelectrical activity of rectus femoris muscle among patients with ACL of knee joint injuries was provided. The comparative index of the average amplitude of the sides (injured and intact) showed 1.15 \pm 0.29 conventional units $(\bar{x} \pm S D)$ for the indicators of vastus intermedius and $1.28 \pm 0.23$ conventional units $(\bar{x} \pm S D)$ for musculus rectus femoris, which indicates a decrease of bioelectrical activity of the rectus femoris muscle of the injured limb.

During the primary examination of the changes in support reactions, which were defined while preforming a balance test on the Gamma Platform, patients demonstrated a body weight shift to the intact limb and an asymmetry of load distribution in lower limbs by difference in loading time (dominant) of the intact and injured limb of $30.65 \pm 12.78 \%(\bar{x} \pm S D)$. The average rate of the dominant loading time (\%) for the injured and intact limbs were $34.4 \pm 2.67 \%(\bar{x} \pm S D)$ and $63.48 \pm 2.59 \%(\bar{x} \pm S D)$, respectively.

Thus, the results analysis in the research of the specific limb functional conditions among the examined patients with knee joint $A C L$ injuries allowed to outline the main set of tasks which should be solved in the process of rehabilitation program development.

Having studied the specifics of the lower limb functional conditions among the participants, we described and developed a physical rehabilitation program for patients after ACL reconstruction with arthroscopic operative interventions.

The program consisted of five periods: preoperative, early postoperative, late postoperative, functional periods and a period of higher physical activity. The basis of the program was the use of workout modules on the Gamma Platform, remedial gymnastics, and massage with elements of passive workout 
to increase the movement amplitude in the injured joint and post-isometric relaxation, mechanotherapy on a joint workout machine, and keeping to the orthopaedic routine.

The specific feature of the developed program of physical rehabilitation for patients with injured ACL, comparing with the traditional program, was, particularly (except the early postoperative rehabilitation period) the use of workout modules of 'Boat,' 'Ball swing,' 'Sorting balls,' 'Trampoline jumping,' 'Jump rope,' 'Combined' on the Gamma Platform, which enhanced the elimination of loading asymmetry between intact and injured limbs and the faster recovery of the functional abilities of the operated joint.

The 52 study patients underwent the course of recovery treatment in the Institute of Traumatology and Orthopaedics, National Academy of Medical Sciences of Ukraine. To assess the effectiveness of the rehabilitation program, two groups of patients were formed: the main group, who followed the presented authors' program $(n=21)$, and the control group ( $n=31$ ), who underwent the recovery treatment course including remedial gymnastics, classical massage, and physical therapy methods in accordance with the hospital program. The preoperative indicators for the studied parameters among the patients of the groups did not present statistically significant differences $(p<0.05)$. The study of the results, their comparison with the baseline data (registered during the preoperative period, 1-4 weeks before the surgical intervention), and their evaluation were performed before the discharge from the hospital, during the early postoperative period (up to 2 weeks after the surgical intervention), during the late postoperative period (3-16 weeks after the surgical intervention), and in the functional period (17-20 weeks after the surgical intervention) of the recovery treatment.

Further research on defining prognostic indices of orthopaedic complications development among patients with ACL injuries was instigated by the results of the stabilography research during the functional period of rehabilitation, which showed that $100 \%$ of the surveyed patients experienced middle or high degree asymmetry of lower extremity load balancing (data showed different time of loading for the intact and injured limb) $-38.53 \pm 6.74 \%(\bar{x} \pm S D)-$ which evidenced the fact that patients continued overloading the intact limb. Simultaneously, during the functional period of rehabilitation treatment, the time of loading (dominant) (\%) of the intact and injured limbs significantly differed $(p<0.05)$; the results of the intact limb indices exceeded those of the injured limb indices 1.89-3.38 times.

The follow-up study showed significant positive changes resulting from a decrease of the time of loading (dominant) of the intact and injured limb, e.g. during the functional period of rehabilitation treatment, the patients achieved the presurgical level of indices: $38.53 \pm 6.74 \%(\bar{x} \pm S D)(p \leq 0.05)$.

The patients' injured limb indices increased 0.23 times on average in comparison with the pre-surgical indices, while the indices of the intact limb insignificantly decreased $(p>0.05)$.

Consequently, the dysfunction of the patients' limb load balancing in upright posture remained, which leads to a conclusion that medium or high degree asymmetry of lower extremity load balancing cannot be cured without applying special means of physical rehabilitation, such as exercises on the Gamma Platform.

Taking into consideration that the level of the functional condition of the injured limb is regarded as a system of coordinated parameters, we analysed the set of factors which characterized the pain level, resistance ability of femoral muscles during adduction, abduction, bending, and extension of knee joint, ability to equipoise (taking into consideration the difference in the time of loading of the intact and injured limb in the balance test), available amplitude of movements when bending, and indices of interferential electromyography of maximum contraction of rectus and vastus medialis muscle in the injured and intact limbs. We also took into account time differences when loading (dominant) the intact and injured limbs during the balance test, which were registered at the pre-surgical stage, i.e. as the initial degree of asymmetry of lower extremity loading.

The factor analysis allowed us to generalize several most important factors which characterized the functional condition of the injured limb and to define the impact of each factor on the general sample variance. The use of the principal component method resulted in defining four major factors which explain the $80.86 \%$ mutability of the initial data (Table 1 ).

As presented in Table 1, the biggest input into the mutability of total variance indices was provided by the first factor, with its 34.02 share of total variance. The results show

Table 1. Factors that define the functional condition of the injured limb in patients with knee joint anterior cruciate ligament injury during the functional period of rehabilitation treatment $(n=31)$

\begin{tabular}{|c|c|c|c|c|}
\hline Measurements & Factor 1 & Factor 2 & Factor 3 & Factor 4 \\
\hline Time difference in loading (dominant) of intact and injured limb at pre-surgical stage (\%) & 0.05 & 0.15 & -0.92 & 0.12 \\
\hline Time difference in loading (dominant) of intact and injured limb at late post-surgical stage (\%) & -0.79 & -0.13 & -0.07 & 0.35 \\
\hline Thigh muscle resistance during adduction $(\mathrm{H})$ & 0.83 & 0.11 & -0.39 & 0.02 \\
\hline Thigh muscle resistance during abduction $(\mathrm{H})$ & 0.81 & 0.25 & 0.10 & 0.39 \\
\hline Thigh muscle resistance during knee joint bending $(\mathrm{H})$ & 0.81 & 0.06 & 0.39 & 0.31 \\
\hline Thigh muscle resistance during knee joint extension $(\mathrm{H})$ & -0.24 & 0.36 & 0.17 & 0.79 \\
\hline Pain level (mm) & 0.14 & 0.67 & -0.43 & -0.06 \\
\hline Mean amplitude of maximum contracture of vastus medialis muscle of the injured limb (mkV) & -0.55 & 0.72 & 0.29 & -0.09 \\
\hline Mean amplitude of maximum contracture of rectus muscle of the injured limb (mkV) & -0.26 & 0.79 & 0.08 & -0.22 \\
\hline Bending of the injured limb (normally $-140^{\circ}$ ), angle of deviation (degree) & 0.58 & 0.25 & 0.34 & -0.39 \\
\hline Total variance & 3.40 & 1.89 & 1.58 & 1.22 \\
\hline Factor's input in total variance (\%) & 34.02 & 18.9 & 15.79 & 12.15 \\
\hline
\end{tabular}


that the indices of loading asymmetry of the intact and injured limb and the indices of muscle resistance during adduction, abduction, and bending of the knee joint play a key role in the leading of the first factor.

The second factor (18.9\% of total variance) includes indices of interferential electromyography of maximum contraction for the rectus and vastus medialis muscle of the injured and intact limbs.

The third factor, which involves time differences when loading (dominant) the intact and injured limbs at the pre-surgical stage, explains $15.79 \%$ of variance. This factor was identified as the predictor of orthopaedic complications development during the process of rehabilitation treatment, namely, of the loading asymmetry of the intact and injured limb.

The fourth factor brings $12.15 \%$ to the total variance and includes indices which characterize the thigh muscle resistance while bending the knee joint.

\section{Discussion}

Incomplete diagnostics of capsular ligamentous apparatus injuries and subsequent inadequate treatment result in the development of chronic multiplanar instability, rapid progression of the degenerative and dystrophic processes in the joint, which is accompanied by the work decrement and disability of patients [4-6]. Such knee joint injuries result in a disruption of proprioceptive knee joint movement analysis. Instability gradually progresses and the pathologic process involves other passive and active stabilizing structures, which were not damaged earlier, which is accompanied by the development of chronic inflammation and degeneration process in the joint.

The literature analysis shows that the treatment of patients with intrasynovial injuries should be carried out with necessary considerations for anatomic and functional peculiarities, the use of methods of early and full restoration of injured joint structures, and strive for reconstruction surgeries that do not require immobilization of the joint.

Accordingly, there is a possibility to develop uniform rehabilitation programs for surgical procedures of the same type. Individual approach is formed specifically in the process of defining the type of rehabilitation program as a result of the analysis of limitations caused by the general physical condition of the patient, degree of the joint structure injury, the specific character of such injuries removal in the course of the surgical procedure, and requests of the patient of household and office nature, with obligatory complication prevention measures.

Cooper et al. [3] investigated the effect of proprioceptive and balance exercise on outcomes following injury and surgical reconstruction of ACL. Five studies of high quality that offered empirical evidence by comparing one rehabilitation program to another were included in this review. There is some evidence that proprioceptive and balance exercise improves outcomes in individuals with ACL-deficient knees. Improvements in joint position sense, muscle strength, perceived knee joint function, and hop testing were reported following proprioceptive and balance exercise. Only one included study investigated proprioceptive exercise following $A C L$ reconstruction. Benefits were noted in the proprioceptive group for measures of strength and proprioception; however, no benefits were observed for any measures of activity. No detrimental effects - such as increased passive joint laxity or decrease in strength - were reported when compared with standard rehabilitation programs for both ACL-deficient and
ACL-reconstructed individuals. Further research is required to determine if proprioceptive and balance exercise improves long-term outcomes such as return to sport [7, 8].

A study by L'Hermette et al. [9] shows that the ACL laxity thresholds may be useful measures to check the status of the ACL and to suggest full-thickness ACL tears. There is sufficient evidence to warrant the implementation of further control measures, including the development of preventive steps for young elite women handball players, as the repetitive nature of specific manoeuvres can lead to uncommon alterations.

In the majority of cases, ACL injuries require specialist surgical and physiotherapeutic treatment. The fact that the patient regains a full range of flexion and extension in the knee joint, as well as a reduction or elimination of pain is a significant determinant of successful physiotherapy. A quicker recovery of the range of extension in the operated knee joint and lower intensity of pain in the final measurement noted in the research group may indicate a certain therapeutic value of pre-surgical physiotherapy [10].

The result of the agonist/antagonist ratio parameter obtained in the healthy limbs among patients in whom exclusively physiotherapeutic procedures were applied was close to the model ratio between the strength of flexor muscles to extensors of the knee joint and equalled $61.5 \%$. However, for the operated limb, this indicator was $42 \%$. The $19.5 \%$ imbalance of the muscle strength observed in this group of patients indicates the need to enhance the strength of knee joint flexors. In patients who applied individual therapy and physiotherapy, the difference in the agonist/antagonist ratio between the limbs was $4.5 \%$, which is the normal value of muscle strength. No differences in muscle strength were found when the duration of rehabilitation of up to 4 weeks or more than 5 weeks was considered. Nevertheless, there are also results that indicate an increase in the strength of flexors among patients who were rehabilitated for a longer time. Analysis should be performed in a larger group of patients in order to determine the effect of the duration of rehabilitation on knee joint flexors and extensors strength parameters. In many rehabilitation programs, and according to the belief of many therapists, there is a need to focus therapeutic actions on strengthening knee joint flexors after ACL reconstruction, which may be consistent with the results obtained in the presented study [1].

\section{Limitations}

We recognize that our pilot study has some limitations. It would be interesting to perform a prospective study with adults aged $30-40$ or even $40-50$ years to follow the course of joint laxity over time, and the number of participants should be increased to confirm the correctness of the results.

\section{Conclusions}

On the basis of the performed analysis of indices that characterize the functional condition of the injured limb of patients with an injury of ACL of knee joint at the functional stage of rehabilitation treatment, it is possible to identify a high and medium level of loading asymmetry in the lower extremities, decrease of electrobiological activity and muscle resistance ability during bending, stretching, adduction, and abduction, as the main prognostic criterion of orthopaedic complications development. 


\section{Disclosure statement}

No author has any financial interest or received any financial benefit from this research.

\section{Conflict of interest}

The authors state no conflict of interest.

\section{References}

1. Płocki J, Kotela I, Pikuła D, Bejer A, Probachta M, Kotela A. Isokinetic assessment of extensor and flexor muscles of the knee joint in patients after anterior cruciate ligament reconstruction treated by the LARS method preliminary report. Stud Med. 2018;34(2):133-140; doi: 10.5114/ms.2018.76874.

2. Chemiris AY, Davydenko AV. Physical rehabilitation of patients with damage to the anterior cruciate ligament of the knee joint. Chronicle of Traumatology and Orthopedics. 2011;1-2:271.

3. Cooper RL, Taylor NF, Feller JA. A systematic review of the effect of proprioceptive and balance exercises on people with an injured or reconstructed anterior cruciate ligament. Res Sports Med. 2005;13(2):163-178; doi: 10.1080/15438620590956197.

4. Kuznetsov IA. Arthroscopic surgery of knee joint [in Russian]. St. Petersburg: s.n.; 2000.

5. Klimenko GS, Zedhenidze IV, Klimenko IG. Open treatment of recent knee joint injuries [in Russian]. Irkutsk: s.n.; 2016.

6. Koroliov AV. Physical rehabilitation of patients after arthroscopic surgeries on knee joint [in Russian]. Emergency Health Service. 2003;Special edition:48.

7. Nikanorov OK. Application of means of physical rehabilitation for athletes of playing sports after reconstruction of the anterior transmitted ligament (on the example of research of the tone of the quadriceps of the thigh) [in Ukrainian]. The Theory and Method of Physical Education and Sports. 2014;1:61-65.

8. Nikanorov OK. The problem of injuries in the game types of sports and the prospects of using physical means of rehabilitation [in Ukrainian]. Sports Medicine. 2015;1-2: 61-65.

9. L'Hermette M, Coquart J, Senioris A, Chamari K, Tourny C, Dujardin F. Pathological knee laxity in elite women team handball players: a pilot study. Biol Sport. 2018;35(2): 159-164; doi: 10.5114/biolsport.2018.72761.

10. Zduński S, Rongies W, Ziółkowski M, Kozieł T, Kazimierski P, Hałaj R, et al. Assessment of knee joint range of motion and the level of pain in patients after arthroscopic ACL reconstruction in the selected physiotherapy model. AdvRehab.2017;3:41-54;doi:10.1515/rehab-2015-0072. 